\section{Corticosteroids suppress in vitro tonsillar proliferation in children with obstructive sleep apnoea}

\author{
L. Kheirandish-Gozal*, L.D. Serpero*, E. Dayyat*, J. Kim*, J.L. Goldman\# , A. Snow*, \\ R. Bhattacharjee* and D. Gozal*
}

ABSTRACT: Intranasal corticosteroids (CS) are potentially useful interventions for children with obstructive sleep apnoea (OSA), and may reduce lymphadenoid tissue size in the upper airway. The present authors hypothesised that CS would reduce cellular proliferation and the production of pro-inflammatory cytokines in a tonsil/adenoid mixed-cell culture system.

Dissociated tonsils or adenoids harvested intra-operatively from children with polysomnographically diagnosed OSA were cultured in control medium (CO) or after stimulation with lipopolysaccharide and concanavalin A (STIM), and incubated with dexamethasone (DEX; $10^{-5}$ $10^{-7} \mathrm{M}$ ), fluticasone (FLU; $10^{-5}-10^{-14} \mathrm{M}$ ) and budesonide (BUD; $10^{-4}-10^{-14} \mathrm{M}$ ). Proliferation and apoptosis were assessed, and supernatants were assayed for the cytokines tumour necrosis factor (TNF)- $\alpha$, interleukin (IL)-6 and IL-8.

STIM increased tonsillar and adenoidal proliferation compared with $C O(1,976 \pm 133$ versus $404 \pm 69$ counts $\left.\cdot \min ^{-1} ; n=54\right)$. DEX, FLU and BUD reduced cellular proliferation rates, and exhibited dose-dependent effects, with the potency being FLU $>$ BUD $>$ DEX ( $n=25$ per group). Conversely, CS increased cellular apoptosis ( $n=20$ per group). Furthermore, TNF- $\alpha$, IL-8 and IL-6 concentrations in the supernatant were increased by STIM, and markedly reduced by all CS ( $n=48$ per group).

Whole tissue cell cultures of adenoids and tonsils provide a useful approach for in vitro assessment of therapeutic efficacy of corticosteroids in the management of lymphadenoid hypertrophy that underlies obstructive sleep apnoea in children.

KEYWORDS: Corticosteroids, cytokines, inflammation, obstructive sleep apnoea, T-lymphocytes, tonsillar hypertrophy

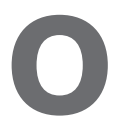
bstructive sleep apnoea (OSA) is a common condition in children, and is characterised by increased upper airway resistance, snoring and partial or complete intermittent obstruction of the upper airway during sleep, leading to episodic oxyhaemoglobin desaturations, hypercapnia and repeated arousals [1]. Adenotonsillar hypertrophy is by far the major contributor to the pathophysiology of OSA in children [2,3], and although a combination of structural and neuromuscular abnormalities contribute to the occurrence of OSA in children, the severity of OSA correlates, albeit loosely, with adenoid and tonsillar size [4-6]. Thus, surgical extirpation, tonsillectomy and adenoidectomy (T\&A), of these tissues is usually the first line of treatment [7].

It has now become apparent that both nasal and oropharyngeal mucosal inflammation are present in children with OSA, and may contribute to increased adenotonsillar proliferation in children with OSA [8]. The recently reported increase in the expression of glucocorticoid receptors in upper airway lymphoid tissues would globally predict favourable outcomes when using intranasal corticosteroids (CS) in paediatric OSA [9], and indeed intranasal CS have shown promising efficacy in reducing the size of the upper airway lymphoid tissues [10-14]. However, the mechanisms underlying the favourable effects of topical CS in inducing the involution of hypertrophic adenoids and tonsils remain unclear.

A novel method was recently developed allowing for in vitro cell culture of tonsils and adenoids derived from children undergoing T\&A [15]. The present authors hypothesised that CS would lead to dose-dependent reductions in cellular proliferation and increased apoptosis in whole tonsillar
AFFILIATIONS

${ }^{*}$ Division of Sleep Medicine and Kosair Children's Hospital Research Institute, Dept of Pediatrics, ${ }^{\#}$ Dept of Surgery, University of Louisville, Louisville, KY, USA.

CORRESPONDENCE

D. Gozal

Dept of Pediatrics,

Comer Children's Hospital,

The University of Chicago, $5721 \mathrm{~S}$. Maryland Avenue, MC 8000, Suite K-160, Chicago, IL 60637, USA.

Fax: 17737024523

E-mail: dgozal@

peds.bsd.uchicago.edu

Received:

August 242008

Accepted after revision:

November 082008

SUPPORT STATEMENT

D. Gozal is supported by the National Institutes of Health (Bethesda, MD,

USA) grants HL-065270, HL-086662 and HL-083075, the Commonwealth of Kentucky Research Challenge for Excellence Trust Fund (Lexington, KY, USA) and the Children's Foundation Endowment for Sleep Research

(Louisville, KY, USA).

STATEMENT OF INTEREST A statement of interest for $L$. Kheirandish-Gozal can be found at www.erj.ersjournals.com/misc/ statments.dt 
and adenoid cell cultures obtained from children with OSA, and that these effects would be associated with a decreased production of pro-inflammatory cytokines.

\section{METHODS \\ Subjects}

The current study was approved by the University of Louisville Human Research Committee (Louisville, KY, USA), and informed consent was obtained from the legal caregiver of each participant. Consecutive children who underwent tonsillectomy for OSA were identified before surgery and recruited into the current study. Overnight polysomnography was performed using standard methods that have been published in detail elsewhere [16]. The diagnosis of OSA was defined by the presence of an obstructive apnoea/hypopnoea index $\geqslant 5$ events $\cdot \mathrm{h}^{-1}$ of total sleep time in the presence of habitual snoring in otherwise healthy children without any disorders requiring treatment with medications, including inhaled or oral CS, leukotriene modifiers, antibiotics or anti-histaminics, or with any known genetic or craniofacial syndrome.

\section{Cell culture}

Surgically removed tonsils and adenoids were placed in ice cold PBS plus antibiotics and processing was started within $30 \mathrm{~min}$ after surgical excision under aseptic conditions. Briefly, tonsils were washed thoroughly with PBS, manually dissected into petri dishes, and gently grounded with a syringe plunger through a $70-\mu \mathrm{m}$ mesh screen to obtain a mixed cell suspension through mechanical dissociation. Red blood cells were removed using a lysis buffer. Cell viability of all specimens was determined by trypan blue exclusion. Specimens with a viability of $<70 \%$ were discarded. Cell cultures were established in standard RPMI 1640 medium supplemented with $10 \%$ heat-inactivated foetal bovine serum (FBS) plus antibiotics, which included streptomycin, fungisone, gentamycin and penicillin to prevent bacterial and fungal contamination. Mixed cell suspensions were transferred onto 96-round bottom-well plates at a concentration of $1 \times 10^{6}$ cells $\cdot$ well $^{-1}$. Cells were cultured in a $5 \% \mathrm{CO}_{2}$ incubator at $37^{\circ} \mathrm{C}$ for $48 \mathrm{~h}$. Cells were also cultured using 24-well plates to determine proinflammatory cytokine levels. Cultures were either maintained as described (control medium (CO)) or stimulated with $25 \mu \mathrm{g} \cdot \mathrm{mL}^{-1}$ lipopolysaccharide (LPS) plus $10 \mu \mathrm{g} \cdot \mathrm{mL}^{-1}$ concanavalin $\mathrm{A}$ (conA) to test proliferation under stimulated conditions (STIM). LPS from Escherichia coli 055:B5 and conA from Canavalia ensiformis were purchased from Sigma Chemical Co. (St Louis, MO, USA).

CS were added to the medium $24 \mathrm{~h}$ after plating to achieve final concentrations ranging from $10^{-5}$ to $10^{-7} \mathrm{M}$ for dexamethasone (DEX) and $10^{-5}$ to $10^{-14} \mathrm{M}$ for fluticasone (FLU) or budesonide (BUD), all of which were purchased from SigmaAldrich Co. (St Louis).

\section{Proliferation assay}

Cells were incubated for the final $18-20 \mathrm{~h}$ with $0.0185 \mathrm{MBq}$ $(0.5 \mu \mathrm{Ci}){ }^{3} \mathrm{H}$-thymidine in complete medium (Amersham Biosciences, Little Chalfont, UK). Cells were then harvested onto glass-fibre filters with a cell harvester, and radioactivity was measured in a liquid scintillation counter. All experimental conditions were always performed in triplicate, and
${ }^{3} \mathrm{H}$-thymidine uptake results were expressed as the average of the three wells in counts per min.

\section{Bromodeoxyuridine cell proliferation and apoptosis annexin $V$ assays with flow cytometry}

To detect $\mathrm{T}$ - and B-cell specific proliferation, the present authors employed bromodeoxyuridine (BrdU) pulsed proliferation analysis using flow cytometry. All procedures were measured using the APC BrdU flow kit (BD Biosciences, San Diego, CA, USA) as recommended by the manufacturer. In brief, at the end of $48 \mathrm{~h}$ of cell culture in 24-well plates, cells were pulse-labelled with $1 \mathrm{mM}$ BrdU for $4 \mathrm{~h}$. The cells were then washed with PBS, and BrdU labelled cells were stained with a three-colour antibody combination consisting of mouse anti-human CD45/PerCP Cy7, CD3/PE and CD19/APC-Cy7 antibodies (BD Biosciences) in $50 \mu \mathrm{L}$ staining buffer for $15 \mathrm{~min}$ on ice. Following binding, with the cell-surface antibodies, cells were fixed and permeabilised with cytofix/cytoperm buffer. After this procedure, cells were suspended with DNase $\left(300 \mu \mathrm{g} \cdot \mathrm{mL}^{-1}\right)$ for $1 \mathrm{~h}$ at $37^{\circ} \mathrm{C}$. The anti-BrdU APC antibody was added in perm/wash buffer and incubated for $20 \mathrm{~min}$ at room temperature. Isotype controls relevant for each antibody were used to establish background fluorescence. A negative control was used as a sample, which was untreated with BrdU and was not stained with specific fluorescence antibodies. Data were acquired on a FACSAria flow cytometer using the FACSDiva 5.5 software (BD Biosciences). After gating of lymphocytes based on CD45+ cells, $\mathrm{T}$ - and B-cell numbers were calculated as CD3+/CD19- and CD3-/CD19+ cell populations, respectively. Moreover, proliferation of $\mathrm{T}$ - and B-cells was identified by counting CD3+/BrdU+ and CD19+/ BrdU+ cell populations. A similar approach was undertaken using annexin $\mathrm{V}$ mouse anti-human antibodies to quantify T- or B-cell specific apoptosis. The results were displayed as two colour dot-plots and analysed by FlowJo software (Tree Star, San Carlos, CA, USA). All data are expressed as the percentage of positive cell from the total cell population.

\section{Apoptosis assay}

Cellular apoptosis was quantified using a cell death detection ELISA kit (Roche Diagnostics, Indianapolis, IN, USA). This immunoassay specifically detects the histone region (H1, H2A, $\mathrm{H} 2 \mathrm{~B}, \mathrm{H} 3$ and $\mathrm{H} 4$ ) of mono- and oligonucleosomes that are released during apoptosis. Apoptosis was measured in duplicate from each treatment group and expressed as absorbance ratios of the CS-treated cell lysates versus absorbances calculated from controls, the latter arbitrarily set at 1.0. The detection limit for this ELISA is $10^{2}$ apoptotic cells.

\section{Cytokine assays}

Concentrations of tumour necrosis factor (TNF)- $\alpha$, interleukin (IL)-8 and IL-6 were measured using commercially available ELISA kits from the supernatants of either CO or STIM conditions. To determine cytokine production, cells were incubated in 24-well flat-bottom plates in complete RPMI medium supplemented with $10 \%$ FBS with or without the addition of $25 \mu \mathrm{g} \cdot \mathrm{mL}^{-1}$ LPS plus $10 \mu \mathrm{g} \cdot \mathrm{mL}^{-1}$ conA. Supernatants were collected after $48 \mathrm{~h}$, and stored at $-80^{\circ} \mathrm{C}$ until assay. TNF- $\alpha$ levels were measured according to manufacturer's instructions, using a high-sensitivity ELISA assay able to detect concentrations as low as $0.09 \mathrm{pg} \cdot \mathrm{mL}^{-1}$ 


\begin{tabular}{|c|c|c|}
\hline TABLE 1 & \multicolumn{2}{|c|}{$\begin{array}{l}\text { Demographic and polysomnographic } \\
\text { characteristics in } 86 \text { children with obstructive } \\
\text { sleep apnoea undergoing tonsillectomy and } \\
\text { adenoidectomy }\end{array}$} \\
\hline Subjects n & & 86 \\
\hline Age yrs & & $6.3 \pm 0.4$ \\
\hline Female \% & & 50 \\
\hline \multicolumn{2}{|c|}{ African-American \% } & 33 \\
\hline \multicolumn{2}{|l|}{ BMI z score } & $1.23 \pm 0.33$ \\
\hline \multicolumn{2}{|l|}{ AHI $\cdot h^{-1}$ TST } & $10.7 \pm 1.9$ \\
\hline \multicolumn{2}{|c|}{ Minimum $\mathrm{Sa}, \mathrm{O}_{2} \%$} & $83.1 \pm 3.6$ \\
\hline \multicolumn{2}{|c|}{ Arousal index $\mathrm{h}^{-1}$ TST } & $17.9 \pm 4.6$ \\
\hline
\end{tabular}

(BioSource Europe SA, Nivelles, Belgium). IL-8 was evaluated using a commercial ELISA kit (R\&D Systems, Minneapolis, $\mathrm{MN}$, USA) with a detection range between 0 and $2,000 \mathrm{pg} \cdot \mathrm{mL}^{-1}$. To determine IL-6 concentrations the IL-6 EASIA assay (BioSource Europe S.A.) was used. The concentrations of cytokines in the supernatants were normalised to the number of cells plated, and expressed as pg. $10^{6}$ cells. For all assays, calibration curves were performed in duplicate for each experiment.

\section{Statistical analysis}

All data were expressed as mean \pm SE, unless stated otherwise. For normally distributed data, ANOVA, followed by post hoc tests, were used, which included Bonferroni correction. Cytokine data were not normally distributed, and were therefore log-transformed and compared using nonparametric tests (Mann-Whitney). To determine whether significant differences in potency occurred among the three CS, multivariate ANOVA with Scheffe post hoc tests were used. All p-values reported are two-tailed with statistical significance set at $<0.05$.

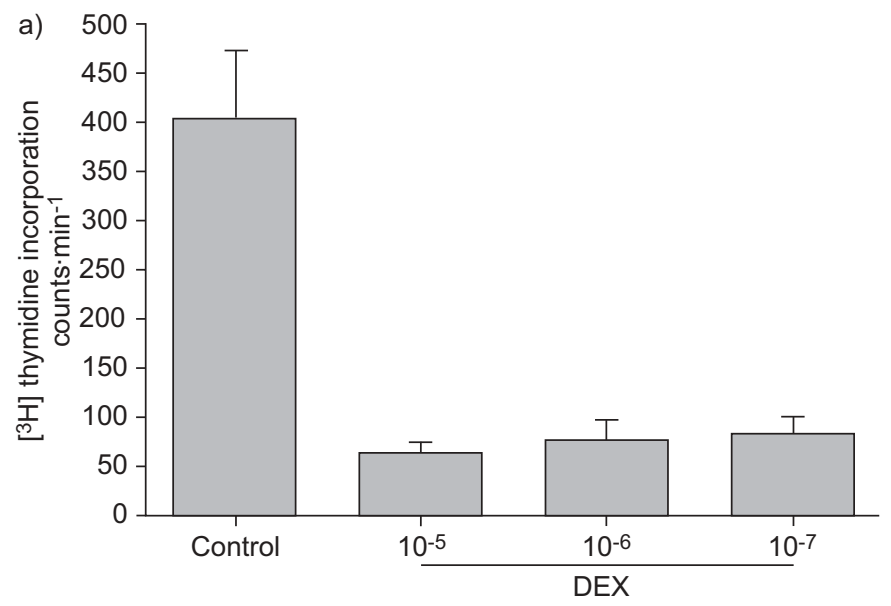

\section{RESULTS}

\section{Study population}

A total of 86 children out of 94 suitable candidates with a clinical and polysomnographic diagnosis of OSA undergoing T\&A agreed to participate and completed the study. The demographic characteristics and major overnight polysomnographic findings for the group are shown in table 1. Of note, due to tissue availability constraints, only a limited number of experimental conditions were possible for every recruited patient, such that the number of subjects included in each experiment varied, and is indicated as appropriate.

\section{Cell proliferation assay}

Basal proliferative rates for mixed cell cultures derived from either tonsils or adenoids were significantly higher after stimulation with LPS and conA (basal conditions: $404.2 \pm 69.0$ versus STIM 1976.3 $\pm 133.1 \mathrm{cpm} ; \mathrm{n}=54 ; \mathrm{p}<0.00001$; fig. 1). Because both the pattern and magnitude or responses were similar between tonsils and adenoids, only combined findings are reported.

To assess the effect of CS on proliferation, initial experiments were conducted using DEX in both CO and STIM conditions. Figure 1 shows the dose-response to incremental doses of DEX, whereby a significant reduction $(\sim 50 \%)$ in proliferation was already apparent at $10^{-7} \mathrm{M}$ compared with control conditions, either without (fig. 1a) or following STIM (fig. 1b). Reductions in proliferation also occurred with either BUD ( $n=18$; fig. 2$)$ or FLU ( $n=18$; fig. 3$)$, except that the concentrations required to achieve $\sim 50 \%$ reduction in proliferation were markedly lower for FLU $\left(10^{-14} \mathrm{M}\right)$ and BUD $\left(10^{-12} \mathrm{M}\right)$ when compared with DEX $(\mathrm{p}<0.0001$ for BUD and FLU versus DEX; figs 1-3). In addition, FLU was more potent than BUD in reducing cellular proliferation by an average of one logarithmic order of magnitude (figs 2 and 3). Indeed, multivariate ANOVA revealed dose-dependent effects, with significant differences in potency as follows: FLU $>$ BUD $>$ DEX $(p<0.0001$; $\mathrm{n}=25$ per group)

To assess whether T- and B-cells are lymphocyte cell subtypes that may be affected by CS, a limited number of experiments $(n=4)$ were performed in which cell cultures were pulsed with

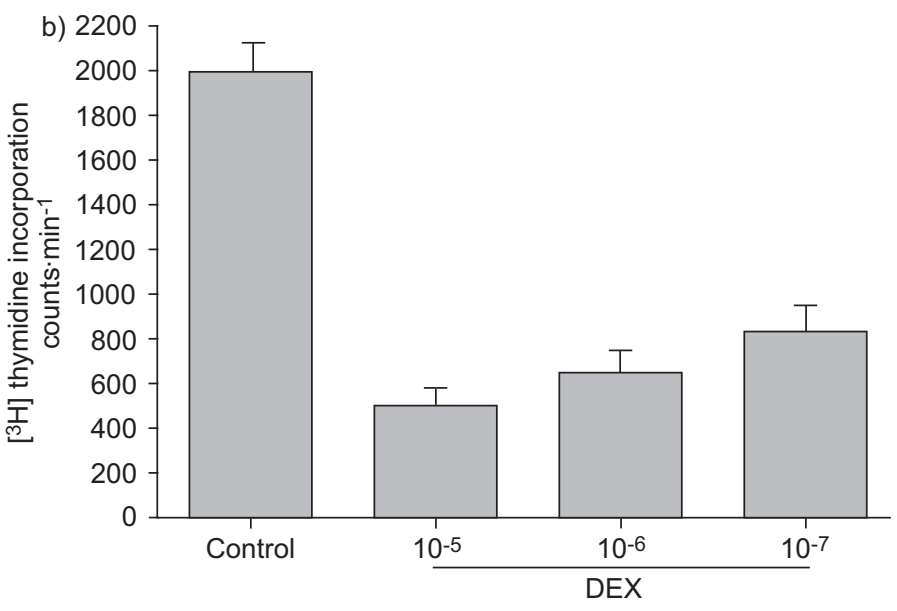

FIGURE 1. Effects of various concentrations of dexamethasone (DEX) on tonsillar cellular proliferation in children with obstructive sleep apnoea in a mixed cell culture system under a) basal conditions or b) after stimulation with lipopolysaccharide and concanavalin $\mathrm{A}$. 

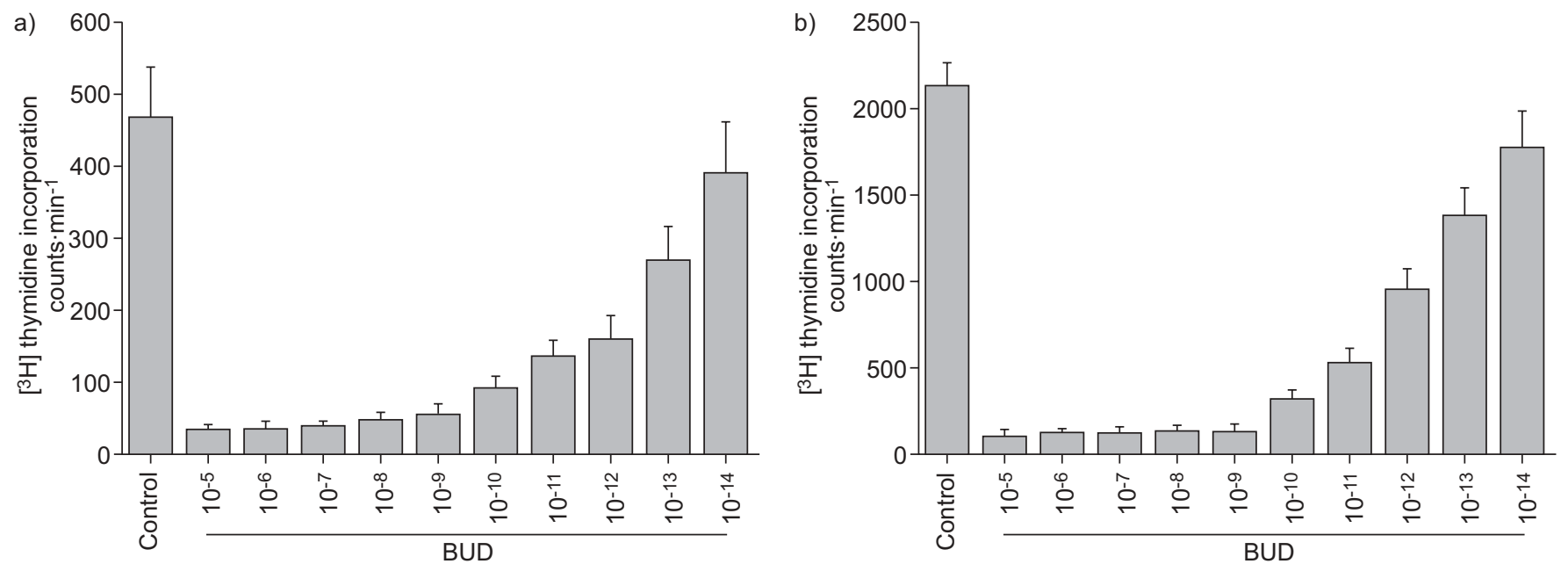

FIGURE 2. Effects of various concentrations of budesonide (BUD) on tonsillar cellular proliferation in children with obstructive sleep apnoea in a mixed cell culture system under a) basal conditions or b) after stimulation with lipopolysaccharide and concanavalin A.

BrdU, after which flow cytometry was employed using either T- or B-cell markers. These experiments confirmed that both CD3+ cells (fig. 4a) and CD19+ cells (fig. 5a) incorporated BrdU, thereby indicating the presence of cellular proliferation. Furthermore, FLU reduced BrdU incorporation in both T(fig. 4) and B-cells (fig. 5), thereby illustrating the antiproliferative effect of FLU on lymphocyte proliferation.

\section{Apoptosis assays}

Cellular apoptosis was only assessed in control unstimulated cell culture conditions. Evidence for increased apoptosis occurred in the presence of all CS at both the maximal dose and at the dose associated with $\sim 50 \%$ reduction in proliferation. Depending on the CS, the mean magnitude of apoptosis was 3.8-6.70-fold that of control conditions at the maximal dose $\left(10^{-5} \mathrm{M}\right.$ for DEX and $10^{-8} \mathrm{M}$ for FLU and BUD; $\mathrm{n}=20$ per group; fig. 6), and around 2.5-3.9-fold at the dose associated with $\sim 50 \%$ reduction in proliferation $\left(10^{-7} \mathrm{M}\right.$ for DEX, $10^{-12} \mathrm{M}$ for FLU and $10^{-13} \mathrm{M}$ for BUD; $\mathrm{n}=20$ per group; fig. 6).
Using annexin $\mathrm{V}$ antibodies and the flow cytometric approach, the present authors found that FLU induced increased apoptosis in both T- (fig. 4c and d) and B-cells (fig. 5c and d) from a mixed cell culture system of tonsils derived from paediatric OSA patients. These findings were consistently observed in three different subjects.

\section{Cytokine assays}

Basal release of TNF- $\alpha$, IL- 8 and IL- 6 to the supernatants was increased in tonsillar cultures from children with OSA after STIM ( $n=48$ per group; fig. 7 ). The addition of DEX at $10^{-7} \mathrm{M}$ (or BUD at $10^{-12} \mathrm{M}$; data not shown) induced significant reductions in pro-inflammatory cytokine production $(\mathrm{p}<0.001$; fig. 7 ).

\section{DISCUSSION}

The present study shows that the stimulation of tonsils in a dissociated mixed cell culture system induces increased proliferative responses and the release of pro-inflammatory cytokines. More importantly, treatment with any of the CS not
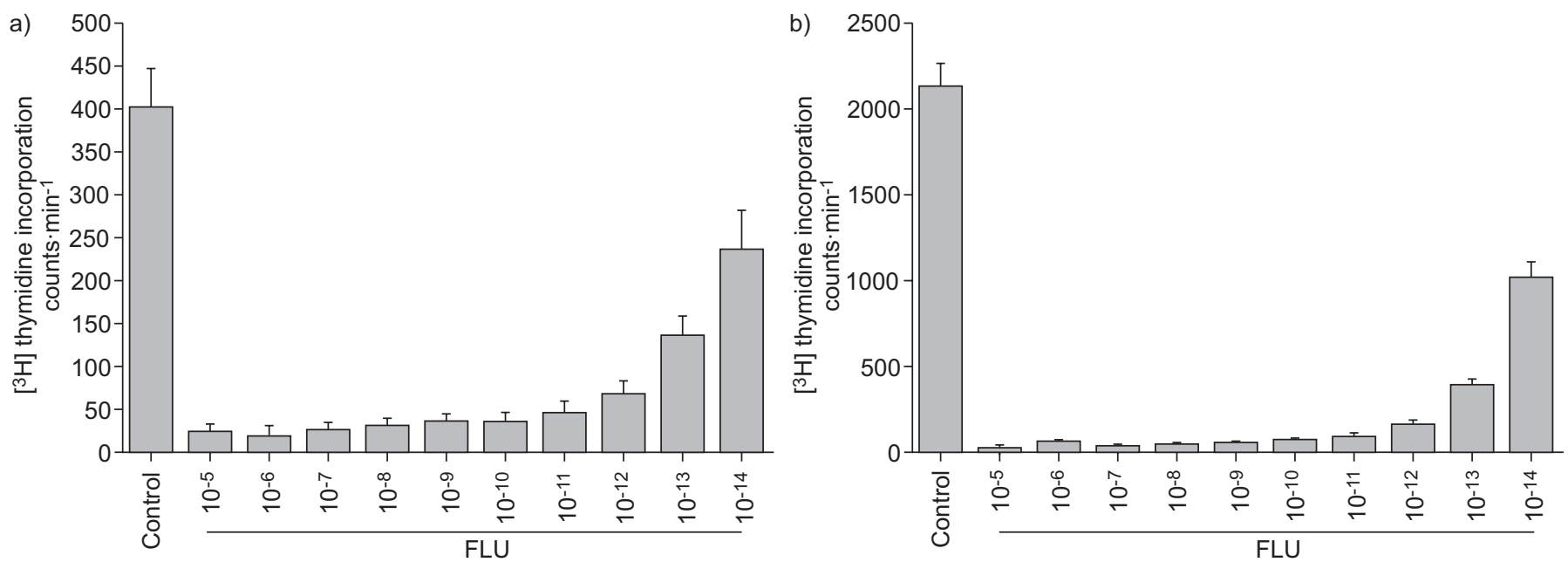

FIGURE 3. Effects of various concentrations of fluticasone (FLU) on tonsillar cellular proliferation in children with obstructive sleep apnoea in a mixed cell culture system under a) basal conditions or b) after stimulation with lipopolysaccharide and concanavalin $\mathrm{A}$. 
only markedly reduces proliferation in a dose-dependent fashion under both control and stimulated conditions, but also reveals striking differences in the respective potencies of DEX, FLU and BUD. The marked decreases in proliferative rates with CS treatment are also accompanied by increased apoptotic cell death, as well as by reductions in the production and release of the pro-inflammatory cytokines TNF- $\alpha$, IL- 8 and IL- 6 . Taken together, the current authors would surmise that the beneficial effects of intranasal and topical CS to reduce the severity of sleep-disordered breathing in children may be due to CS-induced involution of the tonsils and adenoids, and that this novel in vitro approach may provide new insights into therapeutic approaches aiming to resolve frequent paediatric diseases associated with adenotonsillar hypertrophy.

Before the present authors discuss the potential implications of the current findings, two technical and methodological comments are needed. First, the paediatric population included here was not specifically and objectively screened for the presence of allergic rhinitis, even though subjects were not receiving any topical or systemic medications at the time of the surgical removal of their adenoids and tonsils. Previous studies have reported a high prevalence of allergic sensitisation in children with OSA [17-19], even if the role of allergic
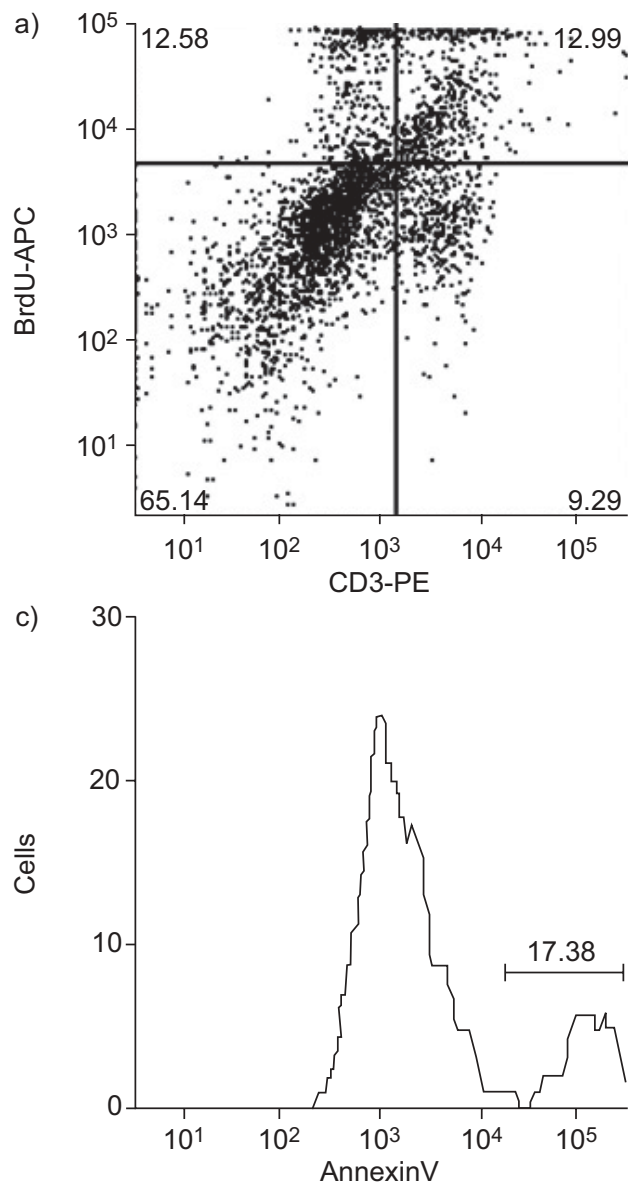

rhinitis in the pathophysiology of OSA remains unclear [20], and despite the similar responses to intranasal CS in mild paediatric OSA, irrespective of allergic history [14]. Notwithstanding such debate, the mixed culture system used herein should allow for exploration of differential responses to established interventions in the presence or absence of an allergic component, as well as in the context of obese children, who traditionally have fared worse after T\&A for OSA [21] Secondly, the selection of LPS and conA as the combined stimulus for the proliferation assays reported herein was based on the lymphocyte populations that reside in tonsillar tissues [22-25]. Indeed, these two substances have marked effects on the predominant populations of immune cell types represented in tonsillar tissues.

The use of topical steroid therapy in paediatric OSA has been the subject of renewed interest, particularly when considering that surgical extirpation of hypertrophic adenoids and tonsils for OSA is accompanied not only by an increased risk for potential post-operative complications and emotional distress for the patient and family, but is also associated with increased health-related costs associated with the surgical procedure. Therefore, studies providing critical assessment of nasal CS in the management of paediatric OSA have been conducted, and have globally produced very encouraging results [10-14].
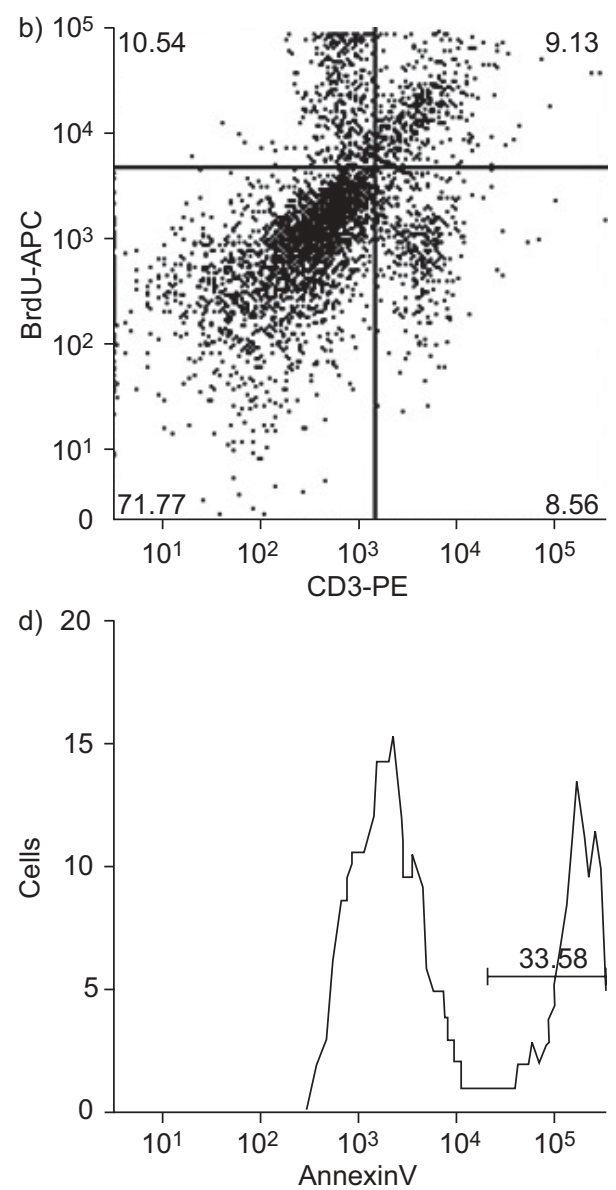

FIGURE 4. Flow cytometry assessment of proliferation of T-lymphocytes (CD3+ cells) in tonsils from a child with obstructive sleep apnoea in a mixed cell culture system in the presence of a) a vehicle or b) fluticasone (FLU $10^{-5} \mathrm{M}$ ). Reduced T-cell proliferation after FLU is apparent. Conversely, T-lymphocyte apoptosis, as assessed by annexin $\mathrm{V}$, and was increased by FLU (d) compared with the vehicle (c). BrdU: bromodeoxyuridine. 

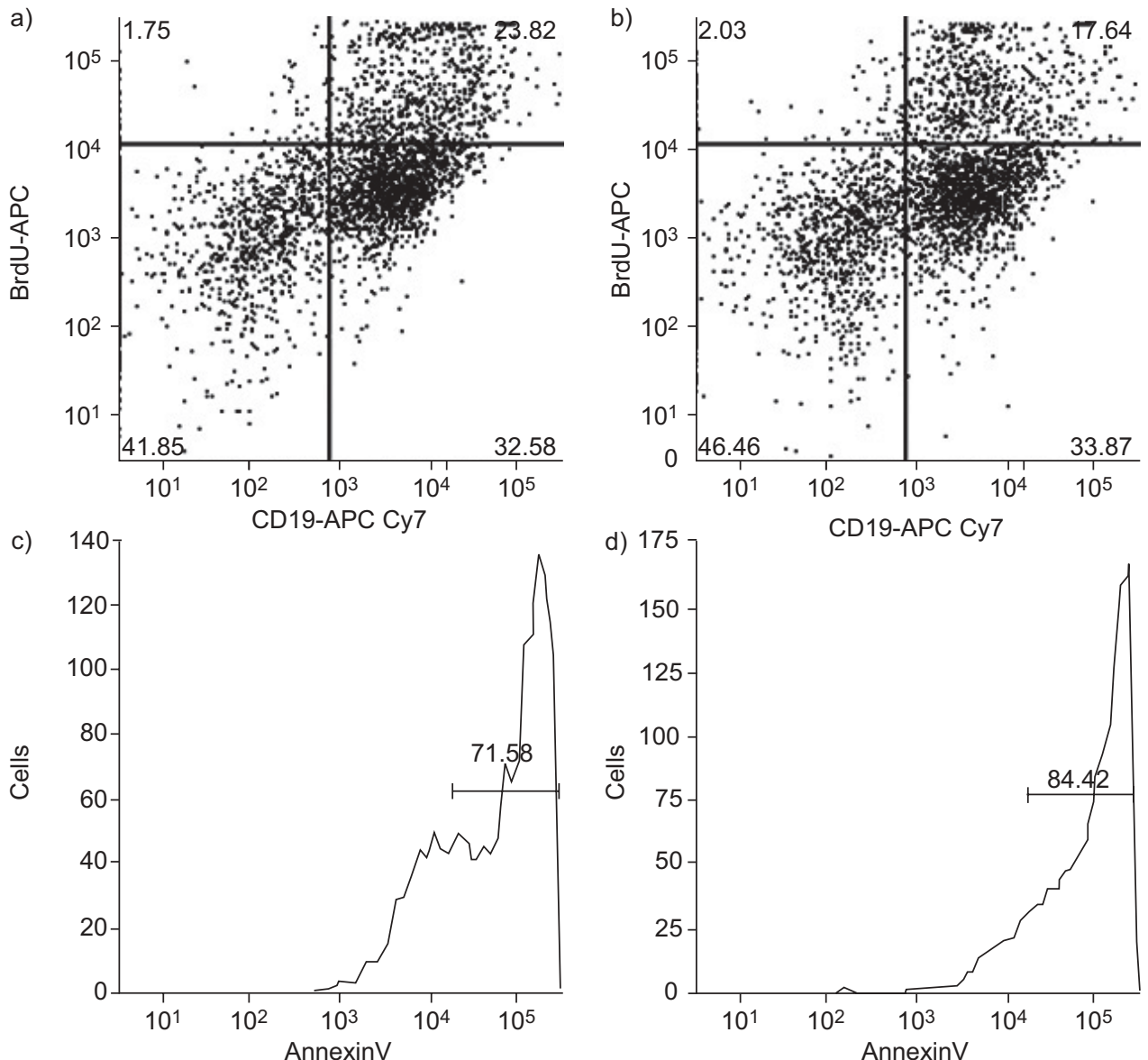

FIGURE 5. Flow cytometry assessment of proliferation of B-lymphocytes (CD19+ cells) in tonsils from a child with obstructive sleep apnoea in a mixed cell culture system in the presence of a) a vehicle or b) fluticasone (FLU $10^{-5} \mathrm{M}$ ). Reduction in B-cell proliferation after FLU is clearly detected. Conversely, B-lymphocyte apoptosis, as assessed by annexin $\mathrm{V}$, was increased by FLU (d) compared with the vehicle (c). BrdU: bromodeoxyuridine.

These studies have shown improved respiratory patterns during sleep across the spectrum of OSA severity. Interestingly, work by AlExOPOULOs et al. [12] and also by

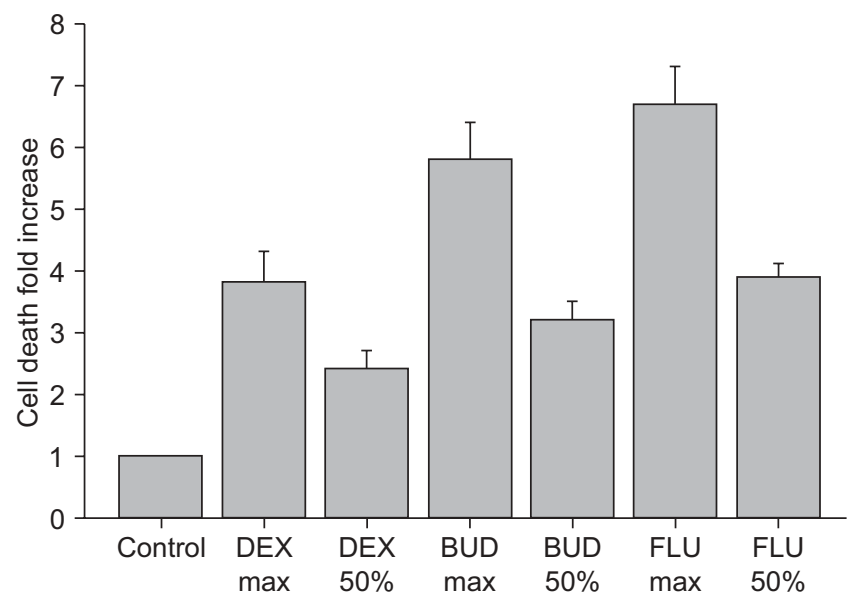

FIGURE 6. Effects of dexmethasone (DEX), budesonide (BUD) and fluticasone (FLU) at maximal concentrations and at concentrations leading to a $50 \%$ reduction of proliferation on tonsillar cellular apoptosis in children with obstructive sleep apnoea in a mixed cell culture system. Results are shown as fold change compared with control conditions (arbitrary value of 1 ).
KHEIRANDISH-GOZAL and GozAL [14] followed the patient cohorts after cessation of treatment with topical CS for up to 9 months, and found evidence for a sustained effect, without rebound increases in symptoms or re-growth of the adenoids and tonsils. However, the optimal dosage and duration of therapy remains undefined, and whether particular groups of patients, such as younger versus older age, or normal weight versus obese would be more likely to favourably respond also remains unknown. Additionally, it remains unclear whether the addition of other anti-inflammatory agents, such as leukotriene receptor antagonists [26, 27], may yield additive or synergistic effects. The in vitro adenotonsillar mixed culture system used in the present study provides the opportunity to examine these issues in great detail, and therefore should allow for delineation and identification of particular sub-groups of patients with OSA, for whom intranasal therapy with CS, for example, would be anticipated to elicit optimal outcomes.

The biological potency of the three CS employed in the current study differed, with FLU having the highest potency in the present mixed cell system proliferation assay. These findings were somewhat anticipated, considering the reported differences in bioaffinity and activity among these compounds [28, 29]. Indeed, FLU is 300 -fold more lipophilic than BUD, has a three-fold higher relative affinity for the glucocorticoid 

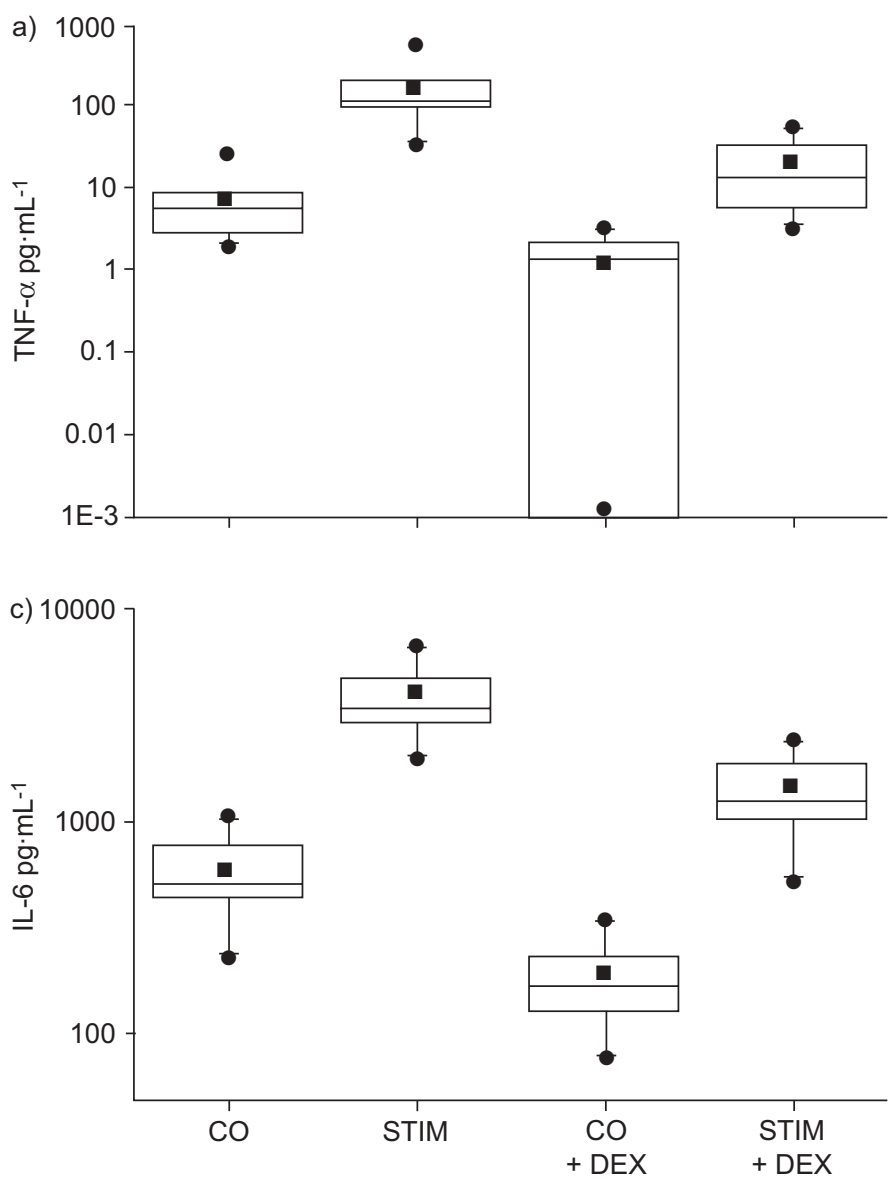

receptor than BUD, and the half-life of the FLU active steroidreceptor complex is $>10 \mathrm{~h}$, compared with $\sim 5 \mathrm{~h}$ for BUD [30]. Similarly, FLU has been found to have increased potency compared with BUD in inhibiting human T-cell migration and proliferation, and inhibiting CD4+ T-cell cytokine release, and in stimulating inflammatory cell apoptosis [30]. Considering the increased abundance of T-cells, particularly CD4+ T-cells, in the tonsillar tissues of children with OSA [31], and the important roles that these cell populations may have in local regulation of immune responses and proliferation [32-34], the use of CS as therapeutic tools seems a logical approach in an attempt to reduce T-cell proliferation and promote apoptosis, while reducing pro-inflammatory cytokine production [35-37]. In the present study, preliminary evidence is presented that both T-and B-lymphocytes proliferate in tonsillar cultures, and that such proliferation can be abrogated by CS, and further elicit increased lymphocyte apoptosis.

In summary, tonsils and adenoids obtained from children with obstructive sleep apnoea undergoing tonsillectomy and adenoidectomy display increased proliferative rates and proinflammatory cytokine production when stimulated with lipopolysaccharide and concanavalin A. Furthermore, treatment with corticosteroids resulted in marked dose-dependent reductions in proliferative rates, increased cellular apoptosis and diminished cytokine release. The relative potency of the three corticosteroids used in the current study was highest for fluticasone and the lowest for dexamethasone. These findings support the use of tonsillar or adenoidal tissue cell cultures as

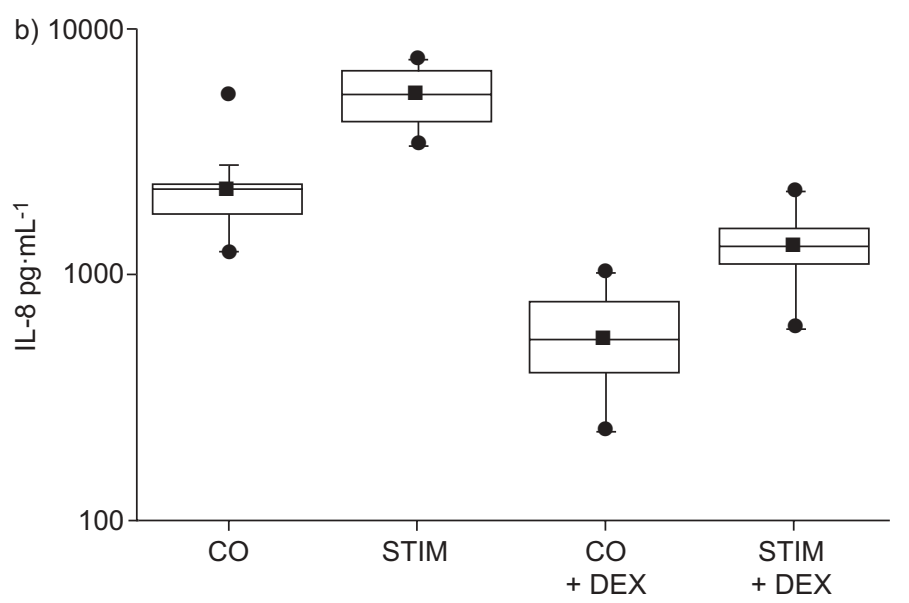

FIGURE 7. Boxplots of concentrations of a) tumour necrosis factor (TNF)- $\alpha, b)$ interleukin (IL)-8 and c) IL-6 in the supernatants of tonsillar cell cultures in basal conditions (CO) or after stimulation with lipopolysaccharide and concanavalin A (STIM) in children with obstructive sleep apnoea, and the effects of dexamethasone (DEX) on the production of these cytokines. - : outliers; $\mathbf{\square}$ : median values.

a potentially useful approach for in vitro assessment of therapeutic efficacy of corticosteroids and other candidate drugs in the treatment of the lymphadenoid hypertrophy that underlies obstructive sleep apnoea in children.

\section{REFERENCES}

1 Lumeng JC, Chervin RD. Epidemiology of pediatric obstructive sleep apnea. Proc Am Thorac Soc 2008; 5: 242-252.

2 Arens R, Marcus CL. Pathophysiology of upper airway obstruction: a developmental perspective. Sleep 2004; 27: 997-1019.

3 Katz ES, D'Ambrosio CM. Pathophysiology of pediatric obstructive sleep apnea. Proc Am Thorac Soc 2008; 5: 253-262.

4 Li AM, Wong E, Kew J, Hui S, Fok TF. Use of tonsil size in the evaluation of obstructive sleep apnoea. Arch Dis Child 2002; 87: 156-159.

5 Lam YY, Chan EY, Ng DK, et al. The correlation among obesity, apnea-hypopnea index, and tonsil size in children. Chest 2006; 130: 1751-1756.

6 Brooks LJ, Stephens BM, Bacevice AM. Adenoid size is related to severity but not the number of episodes of obstructive apnea in children. J Pediatr 1998; 132: 682-686.

7 Schechter MS, Section on pediatrics pulmonology, subcommittee on obstructive sleep apnea syndrome 2002 Technical report. Diagnosis and management of childhood obstructive sleep apnea syndrome. Pediatrics 2002; 109: e69. 
8 Goldbart AD, Krishna J, Li RC, Serpero LD, Gozal D. Inflammatory mediators in exhaled breath condensate of children with obstructive sleep apnea syndrome. Chest 2006; 130: 143-148.

9 Goldbart AD, Veling MC, Goldman JL, Li RC, Brittian KR, Gozal D. Glucocorticoid receptor subunit expression in adenotonsillar tissue of children with obstructive sleep apnea. Pediatr Res 2005; 57: 232-236.

10 Demain JG, Goetz DW. Pediatric adenoidal hypertrophy and nasal airway obstruction: reduction with aqueous nasal beclomethasone. Pediatrics 1995; 95: 355-364.

11 Brouillette RT, Manoukian JJ, Ducharme FM, et al. Efficacy of fluticasone nasal spray for pediatric obstructive sleep apnea. J Pediatr 2001; 138: 838-844.

12 Alexopoulos EI, Kaditis AG, Kalampouka E, et al. Nasal corticosteroids for children with snoring. Pediatr Pulmonol 2004; 38: 161-166.

13 Berlucchi M, Salsi D, Valetti L, Parrinello G, Nicolai P. The role of mometasone furoate aqueous nasal spray in the treatment of adenoidal hypertrophy in the pediatric age group: preliminary results of a prospective, randomized study. Pediatrics 2007; 119: e1392-e1397.

14 Kheirandish-Gozal L, Gozal D. Intranasal budesonide treatment for children with mild obstructive sleep apnea syndrome. Pediatrics 2008; 122: e149-e155.

15 Serpero LD, Kheirandish-Gozal L, Dayyat E, Goldman JL, Kim J, Gozal D. A mixed cell culture model for assessment of proliferation in tonsillar tissues from children with obstructive sleep apnea or recurrent tonsillitis. Laryngoscope 2009; (in press).

16 Montgomery-Downs HE, O'Brien LM, Gulliver TE, Gozal D. Polysomnographic characteristics in normal preschool and early school-aged children. Pediatrics 2006; 117: 741-753.

17 McColley SA, Carroll JL, Curtis S, Loughlin GM, Sampson HA. High prevalence of allergic sensitization in children with habitual snoring and obstructive sleep apnea. Chest 1997; 111: 170-173.

18 Stewart MG. Identification and management of undiagnosed and undertreated allergic rhinitis in adults and children. Clin Exp Allergy 2008; 38: 751-760.

$19 \mathrm{Ng}$ DK, Chan CH, Hwang GY, Chow PY, Kwok KL. A review of the roles of allergic rhinitis in childhood obstructive sleep apnea syndrome. Allergy Asthma Proc 2006; 27: 240-242.

20 Carr E, Obholzer R, Caulfield H. A prospective study to determine the incidence of atopy in children undergoing adenotonsillectomy for obstructive sleep apnea. Int $J$ Pediatr Otorhinolaryngol 2007; 71: 19-22.

21 Tauman R, Gulliver TE, Krishna J, et al. Persistence of obstructive sleep apnea syndrome in children after adenotonsillectomy. J Pediatr 2006; 149: 803-808.

22 Alatas N, Baba F. Proliferating active cells, lymphocyte subsets, and dendritic cells in recurrent tonsillitis: their effect on hypertrophy. Arch Otolaryngol Head Neck Surg 2008; 134: 477-483.
23 Musiatowicz M, Wysocka J, Kasprzycka E, Hassmann E. Lymphocyte subpopulations in hypertrophied adenoid in children. Int J Pediatr Otorhinolaryngol 2001; 59: 7-13.

24 Mansson A, Adner M, Cardell LO. Toll-like receptors in cellular subsets of human tonsil T cells: altered expression during recurrent tonsillitis. Respir Res 2006; 7: 36.

25 Brandtzaeg P. Immunology of tonsils and adenoids: everything the ENT surgeon needs to know. Int J Pediatr Otorhinolaryngol 2003; 67: Suppl. 1, S69-S76.

26 Goldbart AD, Goldman JL, Veling MC, Gozal D. Leukotriene modifier therapy for adenotonsillar hypertrophy and sleep disordered breathing in children. Am J Resp Crit Care Med 2005; 172: 364-370.

27 Kheirandish L, Goldbart AD, Gozal D. Intranasal steroids and oral leukotriene modifier therapy in residual sleepdisordered breathing following tonsillectomy and adenoidectomy in children. Pediatrics 2006; 117: E61-E66.

28 Boorsma $M$, Andersson $\mathrm{N}$, Larsson $\mathrm{P}$, Ullman A. Assessment of the relative systemic potency of inhaled fluticasone and budesonide. Eur Respir J 1996; 9: 1427-1432.

29 Kelly HW. Pharmaceutical characteristics that influence the clinical efficacy of inhaled corticosteroids. Ann Allergy Asthma Immunol 2003; 91: 326-334.

30 Johnson M. Development of fluticasone propionate and comparison with other inhaled corticosteroids. J Allergy Clin Immunol 1998; 101: S434-S439.

31 Kaditis AG, Ioannou MG, Chaidas K, et al. Cysteinyl leukotriene receptors are expressed by tonsillar $\mathrm{T}$ cells of children with obstructive sleep apnea. Chest 2008; 134: 324-331.

32 Johansson-Lindbom B, Ingvarsson S, Borrebaeck CA. Germinal centers regulate human Th2 development. $J$ Immunol 2003; 171: 1657-1666.

33 Park HS, Costalonga M, Reinhardt RL, Dombek PE, Jenkins MK, Cleary PP. Primary induction of CD4 T cell responses in nasal associated lymphoid tissue during group A streptococcal infection. Eur J Immunol 2004; 34: 2843-2853.

34 Yokoi H, Okayama Y, Niyonsaba F, et al. Comparison of human tonsillar mast cell localization and ultrastructural observations between IgE-mediated allergic and nonallergic donors. Allergy Asthma Proc 2006; 27: 415-421.

35 Pajusto $\mathrm{M}$, Thalainen N, Pelkonen J, Tarkkanen J, Mattila PS. Human in vivo-activated CD45R0+ CD4+ T cells are susceptible to spontaneous apoptosis that can be inhibited by the chemokine CXCL12 and IL-2, -6, -7, and -15. Eur J Immunol 2004; 34: 2771-2780.

36 de Baey A, Mende I, Baretton G, et al. A subset of human dendritic cells in the $\mathrm{T}$ cell area of mucosa-associated lymphoid tissue with a high potential to produce TNF- $\alpha$. J Immunol 2003; 170: 5089-5094.

37 Komorowska A, Komorowski J, Banasik M, Lewkowicz P, Tchórzewski H. Cytokines locally produced by lymphocytes removed from the hypertrophic nasopharyngeal and palatine tonsils. Int J Pediatr Otorhinolaryngol 2005; 69: 937-941. 